\title{
Capacitance Response of Concave Well Substrate MEMS Double Touch Mode Capacitive Pressure Sensor: Robust Design, Theoretical Modeling, Numerical Simulation and Performance Comparison
}

Guru Aathavan Alagu Uthaya Kumar

Vellore Institute of Technology: VIT University

Sumit Kumar Jindal ( $\nabla$ sumitjindal08@gmail.com )

VIT University

SREEKANTH P K

Vellore Institute of Technology: VIT University

\section{Research Article}

Keywords: Capacitive pressure sensor, Touch mode, Concave well substrate, Circular Diaphragm, Double touch mode

Posted Date: August 23rd, 2021

DOl: https://doi.org/10.21203/rs.3.rs-825646/v1

License: (9) This work is licensed under a Creative Commons Attribution 4.0 International License. Read Full License

Version of Record: A version of this preprint was published at Silicon on February 4th, 2022. See the published version at https://doi.org/10.1007/s12633-022-01693-9. 


\title{
Capacitance Response of Concave Well Substrate MEMS Double Touch Mode Capacitive Pressure Sensor: Robust Design, Theoretical Modeling, Numerical Simulation and Performance Comparison
}

\author{
Guru Aathavan Alagu Uthaya Kumar, Sumit Kumar Jindal', Sreekanth P K \\ School of Electronics Engineering, VIT University, Vellore, Tamil Nadu, India \\ *Corresponding Author: Email: sumitjindal08@gmail.com, ${ }^{1} \mathrm{Ph}$. No: +91-8603559888
}

\section{Declarations:}

Funding- Not Applicable.

Conflicts of interest/Competing interests -Not Applicable.

Availability of data and material- Not Applicable.

Code availability- Licensed Version of MATLAB has been used to generate plots.

Authors' contributions- Optional (Not Applicable).

\begin{abstract}
Touch Mode Capacitive Pressure Sensor (TMCPS) is very suitable for industrial applications where pressure sensing is necessary because of their linearity, mechanical robust nature and large overload protection from harsh industrial condition. This work proposes an introduction of a notch in the concave substrate for further improvement of the sensitivity of the sensor. Small deflection mode is utilized for the mathematical analysis of the design proposed and MATLAB is utilized for all the software simulations. The sensitivity of the proposed model is very high compared to other models with flat substrate. The analysis and simulation show significant increase in sensitivity in touch mode. The pressure at which the value of the capacitance saturates is also much higher than the designs stated in the literature. The analysis of concave substrate Double Touch Mode Capacitive Pressure Sensor (DTMCPS) will be helpful in designing new sensor for performance increase and to evaluate the behaviour of it.
\end{abstract}

Keywords: Capacitive pressure sensor, Touch mode, Concave well substrate, Circular Diaphragm, Double touch mode.

\section{Introduction}

In the past Decade, the capacitive pressure sensor has been manufactured and used for various applications for its better reliability, low power consumption, small size and ability to integrate along with the low cost of fabrication.

The capacitive pressure sensor is primarily made of two parallel plates with one of them being movable and sensitive to pressure where else the other being fixed and is not sensitive to pressure. So, when the pressure in the environment of the sensor increases the deviation in the diaphragm also increases and so the capacitance of the sensor is increased and the basic analysis is presented in [1]. This is the reason why the capacitive-pressure characteristics is usually nonlinear. A near linear operating region was observed when the diaphragm came in contact with the substrate and the linear characteristic of sensors is presented in [2].

The touch mode pressure sensor has primarily four modes of operation i.e., normal, transition, linear and saturation. The sensor is said to work in normal mode when the diaphragm is not in contact with the substrate. The deflection of the diaphragm can be modelled similarly to that of work presented in [3] and [4]. Further modelling, analysis, and other simulation of flat substrate touch mode sensors are presented in [5][6] about TMCPS before and after touch point.

Several new developments were proposed for the diaphragm and also the structure, and shape of the substrate in order to increase the performance of the sensors. In [7] a double-sided diaphragm was proposed to improve the sensitivity of the sensor and even development in shape of the diaphragm also were introduced to observe the changes in the performance as presented in [8] where a diaphragm with square shape was used, further shape of the substrate was changed to improve performance and reduce strain on the diaphragm by introducing a convex 
shaped diaphragm as presented in [9].Further development in performance was seen with an introduction of a notch in the substrate as the touch point pressure of the sensor increased which is presented in [10] and [11].

The sensor exhibits a linear output only during touch mode. So, to improve the window of linear operation it is important to have a wide range of pressure at which the device operates in touch point. A linear output eliminates the need of complex electronics to process the data from the sensor. Changing the shape of substrate reduced the strain on the diaphragm, where else introduction of a notch not only increased the touch pressure point also increased the sensitivity as presented in [10].

In this work, we propose double touch mode capacitive sensor with the substrate shape being concave and perform its theoretical modelling and simulation of C-P characteristics of the sensor. The sensitivity of the sensor is increased by introduction of a notch in concave well substrate.

\section{Implementation of proposed sensor structure}

The radius of the sphere from which the substrate is sliced is taken to be larger than the radius of the capacitor. The idea of adding a notch like in Fig.1. elongated the linear region of operation. So further adding more notches will further elongate the linear region more. Therefor a concave shaped substrate was designed as in Fig.1 and presented in [12]. The aim of the proposed structure is to observe the changes while adding a notch in concave substrate. The radius of the concave shaped substrate above the notch and in the notch are same.

The capacitance of the proposed design will be higher than that of TMCPS since with introduction of the concave shape the surface area of the substrate is also increased.

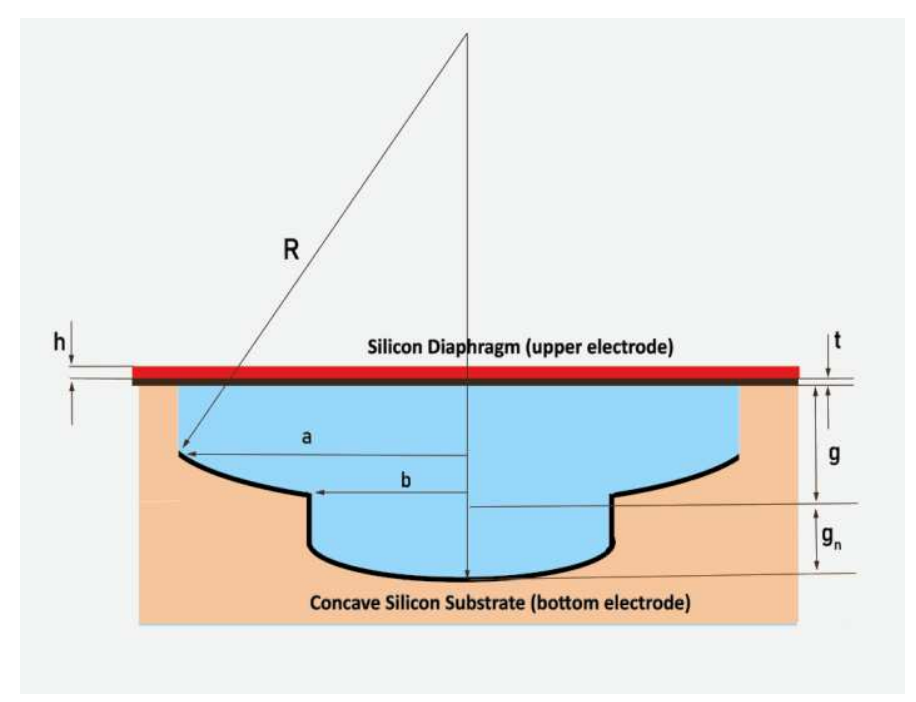

Fig.1. Basic structure of concave substrate double touch mode capacitive pressure sensor (DTMCPS)

$h$ is the thickness of the silicon diaphragm, $t$ is thickness of insulation layer $\left(\mathrm{SiO}_{2}\right), a$ is a radius of silicon diaphragm, $b$ is the radius of the notch, $R$ is radius of sphere and $g_{n}$ is depth of notch.

\section{Mathematical Modelling}

Appling pressure causes the diaphragm to bend towards the substrate and causes the capacitance to increase. The proposed sensor works in normal mode, touch mode and double touch mode. Normal mode is when the diaphragm does not touch the substrate; the diaphragm substrate reaction is as shown in Fig.2. Single touch mode is when the diaphragm touches only the upper part of the substrate as shown in Fig.3 and Fig.4 shows the sensor at first touch point. Double touch mode is when the diaphragm touches both the notch and the upper surface of the substrate as shown in Fig.5.Substrate diaphragm interaction in double touch mode is shown in Fig.6 and Fig.7. 


\subsection{Surface area and touch point pressure}

The surface area of concave well is given by

$$
A=2 \pi R d
$$

The relation between $\mathrm{R}$, $\mathrm{a}$ and $\mathrm{d}$ is given by

$d=R-\sqrt{R^{2}-a^{2}}$

$A=2 \pi R^{2}\left(1-\sqrt{\left(1-\left(\frac{a}{R}\right)^{2}\right)}\right.$

The deflection of circular diagraph is given by Eq. 4

$w(r)=w_{0}\left(1-\left(\frac{r}{a}\right)^{2}\right)^{2}$

Where,

$w_{0}=\frac{P a^{4}}{64 D}$

and $D$ is the flexural rigidity is given by

$D=\frac{E h^{3}}{12\left(1-v^{2}\right)}$

In Eq.5, is the maximum deflection at the centre of the circular diaphragm under the applied pressure $P$. And, in Eq.6 E is Young's Modulus and $v$ is Poisson's Ratio.

\subsection{First touch point}

The deviation of the circular diaphragm at a radius of $b$ should be equal to

$w=g-R+\sqrt{R^{2}-b^{2}}$

And the deviation at $b$ for pressure $P$ is given by

$w=\frac{P a^{4}}{64 D}\left(1-\left(\frac{b}{a}\right)^{2}\right)^{2}$

So, from above equation the pressure of the first touch point can be found

$$
P_{t 1}=\frac{64 D\left(g-R+\sqrt{R^{2}-b^{2}}\right)}{(a-b)^{2}(a+b)^{2}}
$$

The deviation in the diaphragm when the pressure is equal to single touch point pressure $\left(P_{t 1}\right)$ is shown in the Fig.4. 


\subsection{Capacitance for Normal mode of operation}

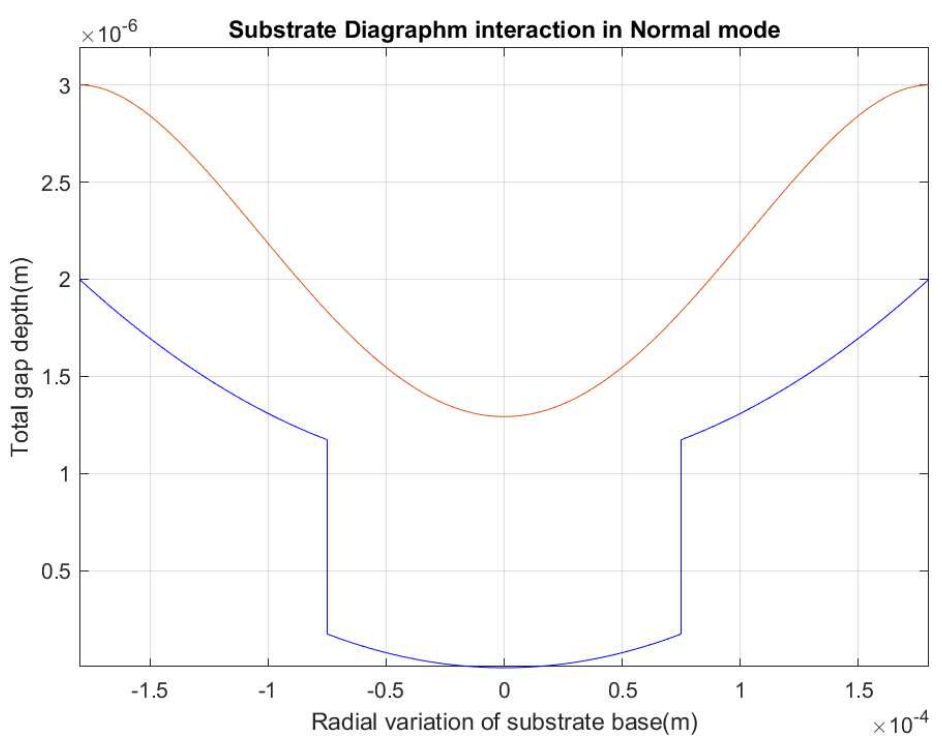

Fig.2. Substrate-diaphragm interaction in normal mode

Where the capacitance of notch area is considered $C_{l}$ and upper concave surface part is considered $C_{2}$

$$
C=C_{1}+C_{2}
$$

And $C_{1}$ and $C_{2}$ are given by

$$
\begin{aligned}
& C_{1}=\int_{0}^{b} \frac{2 \pi \varepsilon_{i} \varepsilon_{0} r d r}{\left(t+\varepsilon_{i}\left(g+g_{n}-w(r)\right)\right)} \\
& C_{2}=\int_{b}^{a} \frac{2 \pi \varepsilon_{i} \varepsilon_{0} r d r}{\left(t+\varepsilon_{i}(g-w(r))\right)}
\end{aligned}
$$

And this relation holds between Capacitance and Pressure until the first touch point. The interaction between the diaphragm and the substrate in touch mode is shown is Fig.3. 


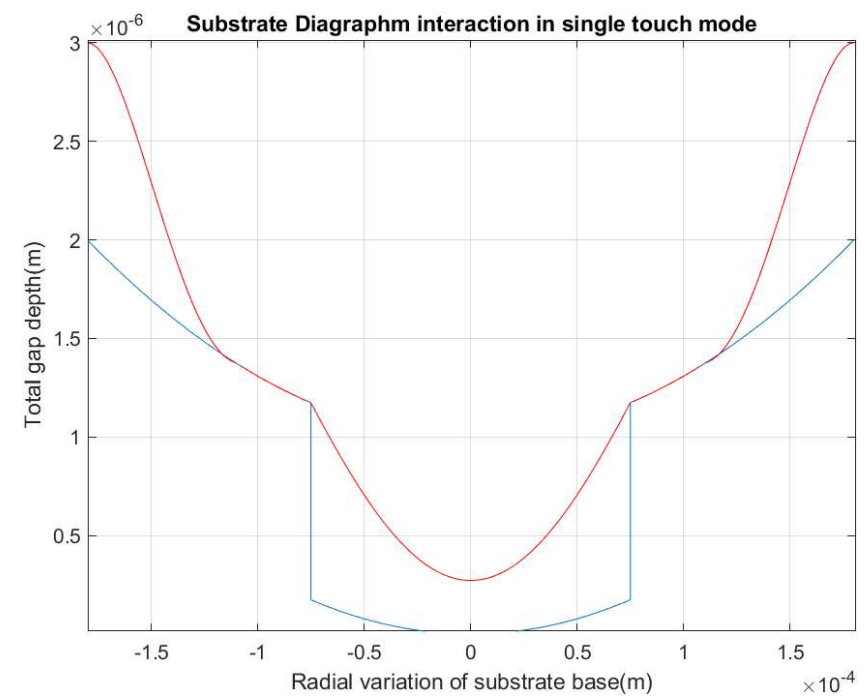

Fig.3. Substrate-diaphragm interaction in Single touch mode

Where

$$
\begin{aligned}
& C=C_{1}+C_{2} \\
& C_{2}=C_{u t 2}+C_{t 2}
\end{aligned}
$$

Here the $C_{2}$ capacitance is split into two parts where $C_{t 2}$ parts denote the part where the diaphragm is in contact with substrate and $C_{u t 2}$ part is not in contact with the substrate.

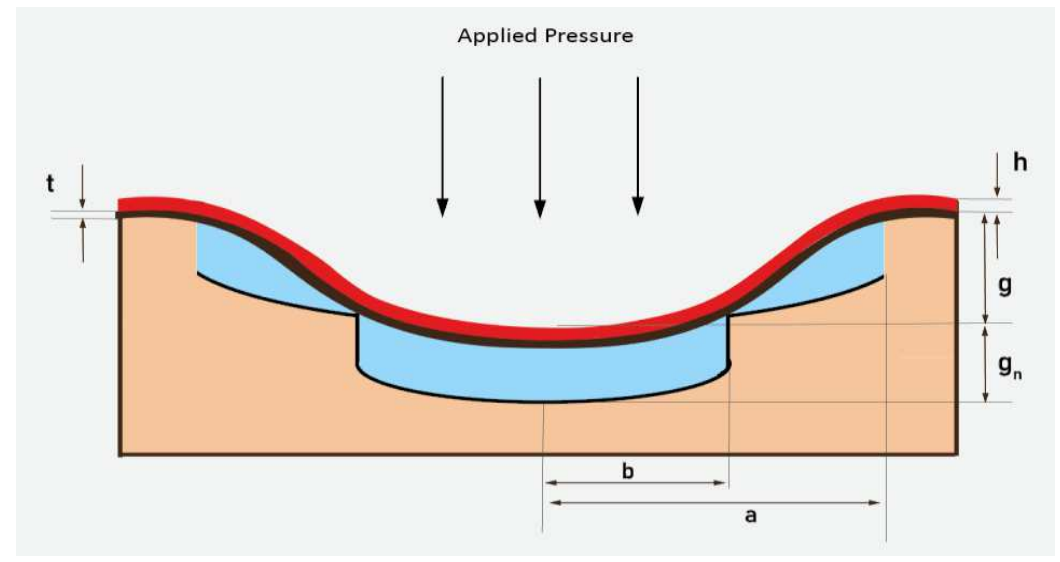

Fig.4. Concave well substrate DTMCPS in single touch mode where the pressure is equal to first touch point pressure 
The deviation of the circular diaphragm for the untouched part can be modelled as

$w(r)=g\left(1-\left(\frac{r-a_{t}}{a-a_{t}}\right)^{2}\right)^{2}$

And the capacitance of untouched part is given as

$$
\begin{aligned}
& C_{u t 2}=2 \pi \varepsilon_{i} \varepsilon_{0} \int_{a_{t}}^{a} \frac{r d r}{\left(t+\varepsilon_{i}(g-w(r))\right)} \\
& C_{u t 2}=2 \pi \varepsilon_{i} \varepsilon_{0} \int_{a_{t}}^{a} \frac{r d r}{t+\varepsilon_{i} g\left(1-\left(1-\left(\frac{r-a_{t}}{a_{u t}}\right)^{2}\right)^{2}\right)}
\end{aligned}
$$

Following substitutions are made in Eq.17

$$
\begin{gathered}
k=\frac{\varepsilon_{i} g}{\varepsilon_{i} g+t} \\
\varphi=\frac{r-a_{t}}{a_{u t}}
\end{gathered}
$$

Therefore, the equations can be written as

$$
C_{u t 2}=\frac{2 \pi \varepsilon_{i} \varepsilon_{0}}{\varepsilon_{i} g+t}\left(a_{u t}^{2} \int_{0}^{1} \frac{\varphi d \varphi}{1-k\left(1-\varphi^{2}\right)^{2}}+a_{u t} a_{t} \int_{0}^{1} \frac{\varphi d \varphi}{1-k\left(1-\varphi^{2}\right)^{2}}\right)
$$

By using two integrals the equations are simplified into

$$
C_{u t 2}=\frac{2 \pi \varepsilon_{i} \varepsilon_{0}}{\varepsilon_{i} g+t}\left(a_{u t}^{2} I_{1}+a_{u t} a_{t} I_{2}\right)
$$

Where $I_{1}$ and $I_{2}$ are given as

$$
\begin{aligned}
& I_{1}=\frac{\arctan h(\sqrt{k})}{2 \sqrt{k}} \\
& I_{2}=\frac{\frac{1}{2} \arctan h\left(\frac{\sqrt{k}}{\sqrt{\sqrt{k}-k}}\right)}{2 \sqrt{\sqrt{k}-k}}+\frac{\frac{1}{2} \arctan h\left(\frac{\sqrt{k}}{\sqrt{\sqrt{k}+k}}\right)}{2 \sqrt{\sqrt{k}+k}}
\end{aligned}
$$




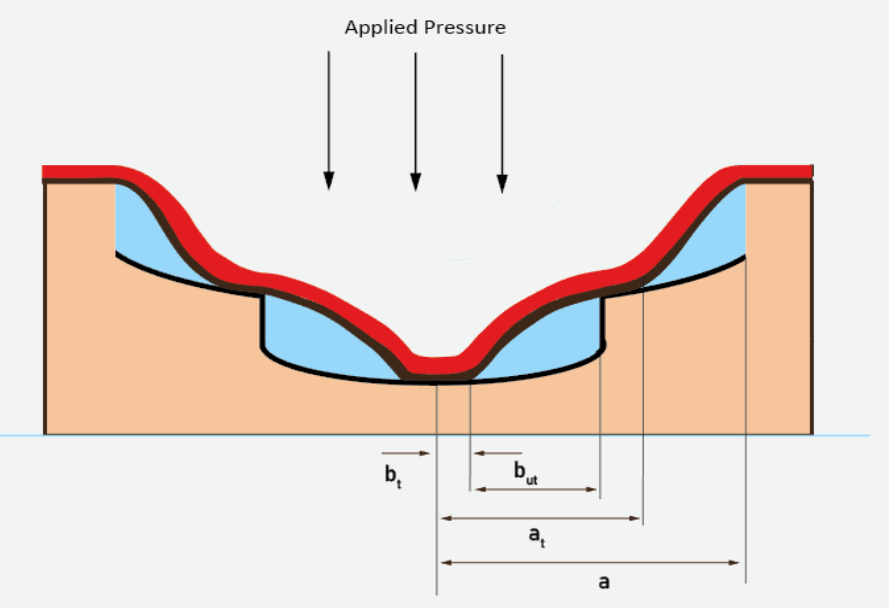

Fig.5. Concave well substrate DTMCPS in double touch mode where the pressure is greater than second touch point

$C_{t}$ for concave substrate with a concave substrate is given as

$C_{t}=\frac{\pi \varepsilon_{i} \varepsilon_{0} \mathrm{R}}{t}\left(1-\sqrt{1-\left(\frac{r}{R}\right)^{2}}\right)$

In case of $C_{2}$, it is calculated as

$C_{t 2}=C_{t}\left(r=a_{t}\right)-C_{t}(r=b)$

$C_{t 2}=\frac{\pi \varepsilon_{i} \varepsilon_{0} \mathrm{R}}{t}\left(\sqrt{1-\left(\frac{b}{a}\right)^{2}}-\sqrt{1-\left(\frac{a_{t}}{a}\right)^{2}}\right)$

And for the $C_{I}$ the diaphragm is supported by the first surface as the deviation increases so deviation is modelled as

$w_{1}(r)=\frac{P_{t 1} a^{4}}{64 D}\left(1-\left(\frac{r}{a}\right)^{2}\right)^{2}+\frac{\left(P-P_{t 1}\right) a^{4}}{64 D}\left(1-\left(\frac{r}{b}\right)^{2}\right)^{2}$

Where the first term models the diaphragm's deflection as it would occur at pressure at $P_{t l}$, and the second term models the further deflection of the diaphragm as supported by the first surface of the concave substrate.

And Capacitance of $C_{l}$ can be found as

$C_{t 1}=\int_{0}^{b} \frac{2 \pi \varepsilon_{i} \varepsilon_{0} r d r}{\left(t+\varepsilon_{i}\left(g+g_{n}-w_{1}(r)\right)\right)}$ 
$C=C_{1}+C_{2}$

$C_{2}=C_{u t 2}+C_{t 2}$

$C_{1}=C_{u t 1}+C_{t 1}$

In this case, $C_{2}$ can modelled similarly as the previous case. $C_{1}$ can be modelled using a similar approach as used in the case of $C_{2}$

In this case $C_{t l}$ can be modelled as

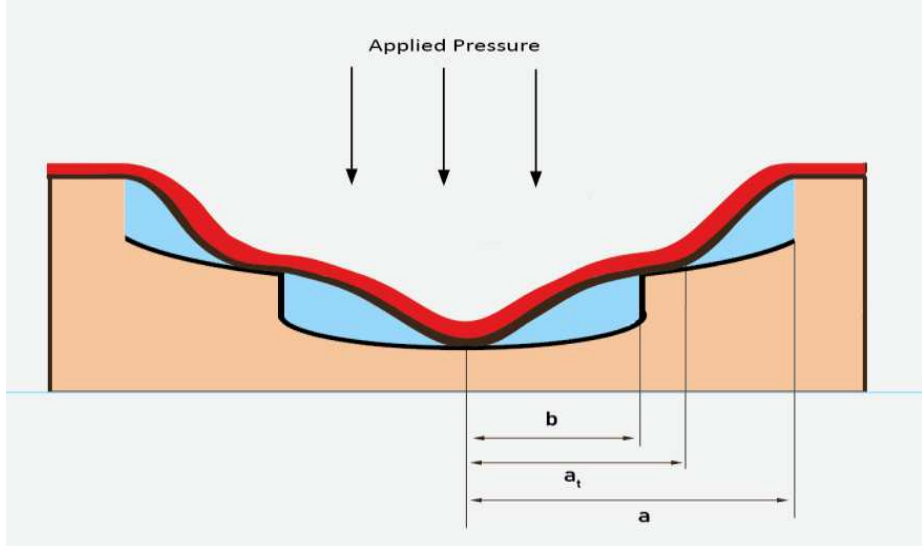

Fig.6. Concave well substrate DTMCPS in double touch mode where the pressure is equal to second touch point

$C_{t 1}=\frac{\pi \varepsilon_{i} \varepsilon_{0} \mathrm{R}}{t}\left(1-\sqrt{1-\left(\frac{b_{t}}{R}\right)^{2}}\right)$

$b_{u t}=b-b_{t}$

$C_{u t 1}=2 \pi \varepsilon_{i} \varepsilon_{0} \int_{a_{t}}^{a} \frac{r d r}{\left(t+\varepsilon_{i}\left(g+g_{n}-w(r)\right)\right)}$

$\frac{P_{t 1} a^{4}}{64 D}\left(1-\left(\frac{r}{a}\right)^{2}\right)^{2} \approx g$ 


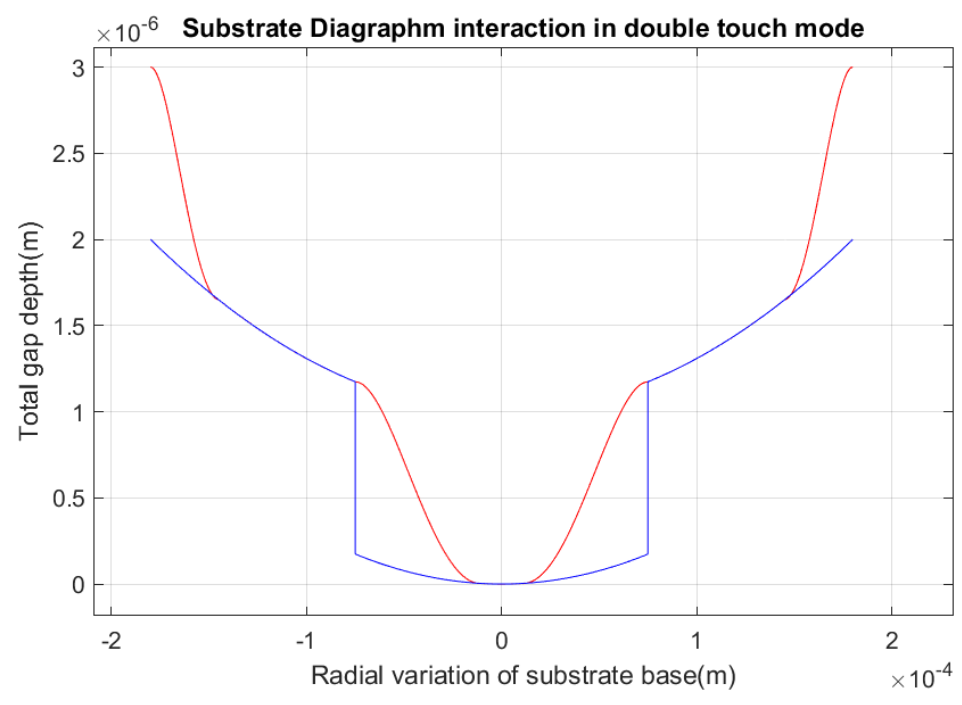

Fig.7. Substrate-diaphragm interaction in Double touch mode

Substituting Eq.35 in Eq.27, we get

$$
\begin{aligned}
& w_{1}(r)=g+g_{n}\left(1-\left(\frac{r-b_{t}}{b-b_{t}}\right)^{2}\right)^{2} \\
& C_{u 1}=2 \pi \varepsilon_{i} \varepsilon_{0}^{b} \int_{b_{t}}^{b} t+\varepsilon_{i} g_{n}\left(1-\left(1-\left(\frac{r-b_{t}}{b_{u t}}\right)^{2}\right)^{2}\right)
\end{aligned}
$$

Following substitutions are made in Eq.37

$$
\begin{aligned}
k^{\prime} & =\frac{\varepsilon_{i} g_{n}}{\varepsilon_{i} g_{n}+t} \\
\varphi^{\prime} & =\frac{r-b_{t}}{b_{u t}}
\end{aligned}
$$

Therefore, the equations can be written as

$$
C_{u t 1}=\frac{2 \pi \varepsilon_{i} \varepsilon_{0}}{\varepsilon_{i} g_{n}+t}\left(b_{u t}^{2} \int_{0}^{1} \frac{\varphi^{\prime} d \varphi^{\prime}}{1-k^{\prime}\left(1-\varphi^{\prime 2}\right)^{2}}+b_{u t} b_{t} \int_{0}^{1} \frac{\varphi^{\prime} d \varphi^{\prime}}{1-k^{\prime}\left(1-\varphi^{\prime 2}\right)^{2}}\right)
$$

By using two integrals the equations are simplified into

$$
C_{u t 1}=\frac{2 \pi \varepsilon_{i} \varepsilon_{0}}{\varepsilon_{i} g_{n}+t}\left(b_{u t}{ }^{2} I_{1}+b_{u t} b_{t} I_{2}\right)
$$


Where $I_{1}$ and $I_{2}$ are given as

$$
\begin{aligned}
& I_{1}=\frac{\arctan h(\sqrt{k})}{2 \sqrt{k}} \\
& I_{2}=\frac{\frac{1}{2} \arctan h\left(\frac{\sqrt{k}}{\sqrt{\sqrt{k}-k}}\right)}{2 \sqrt{\sqrt{k}-k}}+\frac{\frac{1}{2} \arctan h\left(\frac{\sqrt{k}}{\sqrt{\sqrt{k}+k}}\right)}{2 \sqrt{\sqrt{k}+k}}
\end{aligned}
$$

\section{Simulation and Discussion}

The design parameters of concave well substrate DTMCPS are shown in Table 1

Table 1 Design parameters for the proposed design

\begin{tabular}{|l|l|}
\hline Parameter & Design value \\
\hline Pressure Range & $(0-2) \mathrm{MPa}$ \\
Young's modulus $(E)$ & $170 \times 10^{2} \mathrm{~N} / \mathrm{m} 2$ \\
diaphragm thickness $(h)$ & $5 \times 10^{-6} \mathrm{~m}$ \\
Poisson's ratio for silicon $(v)$ & 0.28 \\
Radius of diaphragm $(a)$ & $180 \times 10^{-6} \mathrm{~m}$ \\
Radius of notch $(b)$ & $75 \times 10^{-6} \mathrm{~m}$ \\
Permittivity of vacuum $\left(\varepsilon_{0}\right)$ & $8.854 \times 10^{-12} \mathrm{~F} / \mathrm{m}$ \\
Dielectric constant of $\mathrm{SiO}_{2}\left(\varepsilon_{i}\right)$ & 3.9 \\
Dielectric constant of cavity/air $\left(\varepsilon_{a}\right)$ & 1 \\
Thickness of insulation layer $(t)$ & $0.1 \times 10^{-6} \mathrm{~m}$ \\
Radius of parent sphere $(R)$ & $16200 \times 10^{-6} \mathrm{~m}$ \\
Gap at center of diaphragm $(g)$ & $2 \times 10^{-6} \mathrm{~m}$
\end{tabular}

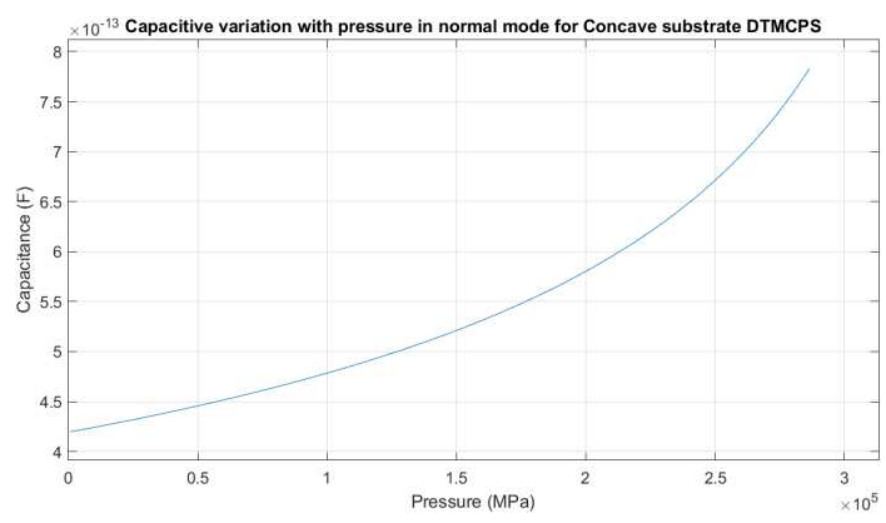

Fig.8. Capacitive variation of concave substrate DTMCPS

The mathematical model derived in order to obtain the relation between pressure and capacitance is used to develop a model. First, the variation of the diaphragm in normal mode is shown in Fig.2. As the pressure increases the deviation of the diaphragm is increases and once the pressure increases more than the first pressure point the diaphragm comes in contact with the first concave surface of the concave substrate which is depicted in Fig.3. As the pressure further increases, the diaphragm wraps more around the concave substrate. Once the pressure reaches more than the second touch point the diaphragm touches the notch area and upon further increase in pressure the diaphragm wraps around the notch area further depicted in Fig.5. 


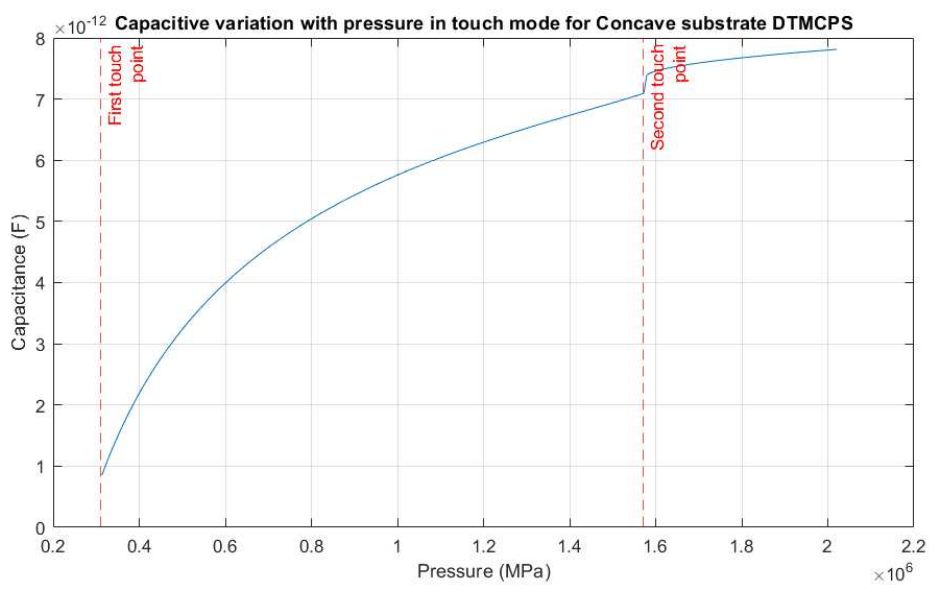

Fig.9.Capacitive variation with pressure in touch mode for concave substrate DTMCPS

The touch point for the above-mentioned design parameter was observed at $0.31 \mathrm{Mpa}$ and the second touch point pressure was observed at $1.57 \mathrm{Mpa}$. The pressure range defined for the normal mode of operation of this design is 0 to $0.31 \mathrm{Mpa}$. And Fig. 8 shows the variation of capacitance with respect to pressure in normal mode.

The touch mode operation starts from a range of $0.31 \mathrm{Mpa}$ and the variation of the capacitance is shown in Fig. 9 and the sensitivity of the curve is shown in Fig.10.

It is clearly depicted from the above result that total touch mode can be observed as two sub regions namely linear and saturation. As saturation is achieved the peak capacitance value is increased up to a value of $8 \mathrm{pF}$.

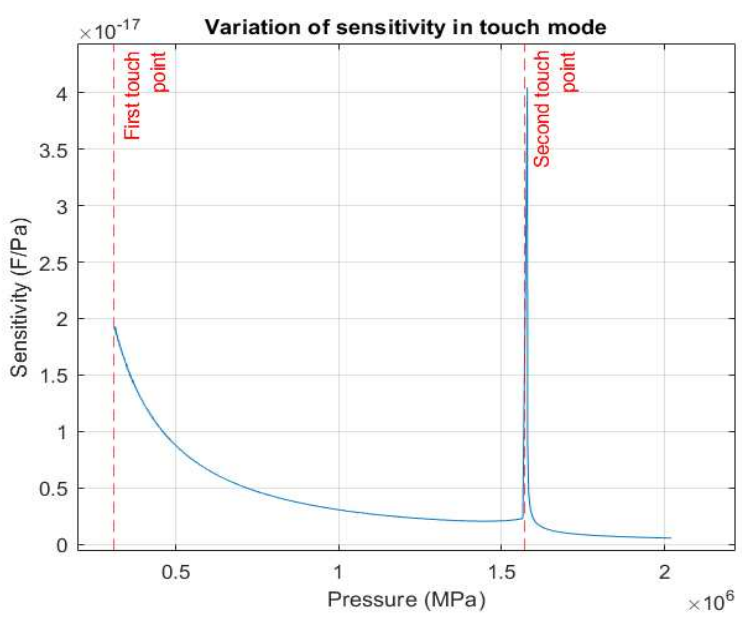

Fig.10.Sensitivity variation with pressure in touch mode 
Table 2 Comparison Table of STMCPS and DTMCPS in touch mode of operation

\begin{tabular}{|c|c|c|c|c|}
\hline Sensor Type & $\operatorname{Size}(\mu \mathrm{m})$ & $\begin{array}{c}\text { Capacitance } \\
\text { developed (pF) for } \\
\text { applied pressure of } \\
1.5 \mathrm{MPa}\end{array}$ & $\begin{array}{c}\text { Capacitance } \\
\text { developed (pF) for } \\
\text { applied pressure of } \\
2 \mathrm{MPa}\end{array}$ & $\begin{array}{l}\text { Comment on } \\
\text { sensitivity }\end{array}$ \\
\hline $\begin{array}{l}\text { STMCPS } \\
\text { (Flat Substrate) [11] }\end{array}$ & $\begin{array}{l}\mathrm{h}=5, \mathrm{a}=180 \text { and } \mathrm{g} \\
=2\end{array}$ & 1.35 & 1.5 & \multirow{4}{*}{$\begin{array}{l}\text { It can be noticed that } \\
\text { in touch mode } \\
\text { operation there is a } \\
\text { significant increase in } \\
\text { sensitivity for the } \\
\text { proposed model }\end{array}$} \\
\hline $\begin{array}{l}\text { DTMCPS } \\
\text { (Flat Substrate) [11] }\end{array}$ & $\begin{array}{l}\mathrm{h}=5, \mathrm{r}=180 \text { and } \mathrm{g} \\
=2\end{array}$ & 2.3 & 2.7 & \\
\hline $\begin{array}{l}\text { STMCPS } \\
\text { (Concave well) [12] }\end{array}$ & $\begin{array}{l}\mathrm{h}=5, \mathrm{r}=180, \mathrm{~g}=2 \text { and } \\
\mathrm{d}=1\end{array}$ & 1.1 & 1.6 & \\
\hline $\begin{array}{l}\text { DTMCPS } \\
\text { (Concavewell, } \\
\text { proposed work) }\end{array}$ & $\begin{array}{l}\mathrm{h}=5, \mathrm{r}=180, \mathrm{~g}=2 \text { and } \\
\mathrm{g}_{\mathrm{n}}=1\end{array}$ & 6.9 & 7.8 & \\
\hline
\end{tabular}

The sensitivity of the proposed model is comparatively high as compared to that of the TMCPS and DTMCPS. Also, the pressure at which the capacitance of the sensor saturates is higher than the other proposed designs(Table 2), improving the working range of the sensor.

\section{Conclusion}

The work deals with an analysis and simulation for DTMCPS with concave well substrate and circular diaphragm. The small deflection model is utilized for mathematical analysis and MATLAB software is utilized for simulation. The concave well substrate DTMCPS shows improved sensitivity and saturation capacitance over the other models. The characteristics of the sensor will depend on the choice of the dimension of the notch because the second touch point plays a key role in enhancing the sensitivity of the device.

\section{AUTHOR DECLARATIONS SECTION}

* Ethics approval and consent to participate: Not Applicable

* Consent for publication: Yes

* Availability of data and materials: Not Applicable

* Competing interests: Not Applicable

* Funding: Not Applicable

* Authors' contributions: All authors contributed to the study conception and design. Material preparation, simulation and analysis were performed by all the authors together. The first draft of the manuscript was written by Mr. Al Guru Aathavan and all authors commented on previous versions of the manuscript. All authors read and approved the final manuscript.

* Acknowledgements: Not Applicable

Compliance with Ethical Standards section

* Disclosure potential conflicts of interest: Not Applicable

* Research involving Human Participants and/or Animals: Not Applicable

* Informed consent: Not Applicable 


\section{Reference}

[1] Meng, Guangqing, and Wen H. Ko. "Modeling of circular diaphragm and spreadsheet solution programming for touch mode capacitive sensors." Sensors and Actuators A: physical 75.1 (1999): 45-52.

[2] Rosengren, Lars, Jan Söderkvist, and Leif Smith. "Micromachined sensor structures with linear capacitive response." Sensors and Actuators A: Physical 31.1-3 (1992): 200-205.

[3] Ding, Xiaoyi, et al. "Touch mode silicon capacitive pressure sensors." ASME Winter Annual Meeting Dallas. Vol. 111. 1990.

[4] Jindal, Sumit Kumar, Ankush Mahajan, and Sanjeev Kumar Raghuwanshi. "A complete analytical model for clamped edge circular diaphragm non-touch and touch mode capacitive pressure sensor." Microsystem Technologies 22.5 (2016): 1143-1150.

[5] Hezarjaribi, Y., and M. N. Hamidon. "Theoretical formulation to evaluate capacitance for before and after touch point MEMS capacitive pressure sensors." Int. J. Eng. Sci. 2.1 (2013): 278-286.

[6] Hezarjaribi, Yadollah, et al. "Evaluation for diaphragm's deflection for touch mode MEMS pressure sensors." Int. Arab J. Inf. Technol. 8.2 (2011): 141-146.

[7] Varma, M. Aditya, Deepali Thukral, and Sumit Kumar Jindal. "Investigation of the influence of doublesided diaphragm on performance of capacitance and sensitivity of touch mode capacitive pressure sensor: numerical modeling and simulation forecasting." Journal of Computational Electronics 16.3 (2017): 987-994.

[8] Han, Xiaodong, et al. "Design and experiment of a touch mode MEMS capacitance vacuum gauge with square diaphragm." Sensors and Actuators A: Physical 313 (2020): 112154.

[9] Varma, M. Aditya, and Sumit Kumar Jindal. "Novel design for performance enhancement of a touch-mode capacitive pressure sensor: theoretical modeling and numerical simulation." Journal of Computational Electronics 17.3 (2018): 1324-1333.

[10] Jindal, Sumit Kumar, M. Aditya Varma, and Deepali Thukral. "Comprehensive assessment of MEMS double touch mode capacitive pressure sensor on utilization of $\mathrm{SiC}$ film as primary sensing element: Mathematical modelling and numerical simulation." Microelectronics Journal 73 (2018): 30-36.

[11] Jindal, Sumit Kumar, and Sanjeev Kumar Raghuwanshi. "Capacitance and sensitivity calculation of double touch mode capacitive pressure sensor: Theoretical modelling and simulation." Microsystem Technologies 23.1 (2017): 135-142.

[12] Kang, MyongChol, Chan Ri, and JongHuan Choe. "Capacitance response of concave well substrate touchmode capacitive pressure sensor: Mathematical analysis and simulation." Microelectronics Journal 114 (2021): 105118 . 